\title{
Regional coal seam gas distribution and burial history of the Hunter Coalfield, Sydney Basin
}

\section{K. L. PINETOWN ${ }^{\dagger}$}

CSIRO Earth Science and Resource Engineering, PO Box 136, North Ryde, NSW, 1670, Australia

\section{SUPPLEMENTARY PAPERS}

Australian Journal of Earth Sciences (2014) 61, http://dx.doi.org/10.1080/08120099.2014.893539

Tables A1-7 listed at the end of the paper are Supplementary Papers; copies may be obtained from the Geological Society of Australia's website (www.gsa.org.au), the Australian Journal of Earth Scienecs website (www.ajes.com.au) or from the National Library of Australia's Pandora archive (http://nla.gov.au/nla.arc-25194).

† Corresponding author:_Kaydy.Pinetown@csiro.au

Table A1 Main model inputs for Knight-1.

Table A2 Main model inputs for Pinegrove-1.

Table A3 Main model inputs for DH219.

Table A4 Main model inputs for DH225.

Table A5 Main model inputs for EHL-1.

Table A6 Main model inputs for EHRP-1.

Table A7 Main model inputs for Wybong- 1 . 
Pinetown 2014 Supplementary Papers

Australian Journal of Earth Sciences 61, doi: 10.1080/08120099.2014.893539

Table 1 Main model inputs for Knight-1.

\begin{tabular}{|c|c|c|c|c|c|c|c|c|c|c|c|c|c|c|c|c|c|}
\hline \multirow[t]{2}{*}{ LAYER } & \multirow{2}{*}{$\begin{array}{l}\text { TOP } \\
\text { (m) }\end{array}$} & \multirow{2}{*}{$\begin{array}{l}\text { BASE } \\
\text { (m) }\end{array}$} & \multirow{2}{*}{$\begin{array}{c}\text { THICKNESS } \\
\text { (m) }\end{array}$} & \multirow{2}{*}{$\begin{array}{c}\text { EROSION } \\
\text { (m) }\end{array}$} & \multirow{2}{*}{$\begin{array}{l}\text { DEPOSITION } \\
\text { FROM } \\
\text { (Ma) }\end{array}$} & \multirow{2}{*}{$\begin{array}{c}\text { DEPOSITION } \\
\text { TO } \\
\text { (Ma) }\end{array}$} & \multirow{2}{*}{$\begin{array}{l}\text { EROSION } \\
\text { FROM } \\
\text { (Ma) }\end{array}$} & \multirow{2}{*}{$\begin{array}{l}\text { EROSION } \\
\text { TO } \\
\text { (Ma) }\end{array}$} & \multicolumn{8}{|c|}{ LITHOLOGY (\%) } & \multirow{2}{*}{$\begin{array}{l}\text { TOC } \\
\text { (\%) }\end{array}$} \\
\hline & & & & & & & & & $\begin{array}{l}\text { CONGLOM } \\
\text { ERATE }\end{array}$ & SANDSTONE & SILTSTONE & MUDSTONE & CLAYSTONE & SHALE & TUFF & COAL & \\
\hline Late Triassic - Jurassic sediments & -97 & -97 & 0 & 1100 & 205 & 135 & 130 & 90 & & 50 & & & & 50 & & & \\
\hline Wianamatta Group & -97 & -97 & 0 & 300 & 215 & 210 & 90 & 80 & & 50 & & & & 50 & & & \\
\hline Hawkesbury Sandstone & -97 & -97 & 0 & 250 & 225 & 215 & 80 & 70 & & 100 & & & & & & & \\
\hline Narrabeen Group & -97 & -97 & 0 & 800 & 252 & 225 & 70 & 4 & 50 & 50 & & & & & & & \\
\hline NCL CM Moon Island Beach Formatior & -97 & -97 & 0 & 48 & 253 & 252 & 4 & 3 & 40 & 30 & 10 & 17 & & & & 3 & 2 \\
\hline NCL CM Boolaroo Formation & -97 & -97 & 0 & 70 & 254 & 253 & 3 & 1 & & 40 & 10 & 23 & & & 10 & 17 & 15 \\
\hline NCL CM Adamstown Formation & -97 & -66 & 31 & 50 & 255 & 254 & 1 & 0 & & 45 & 15 & 28 & & & 5 & 7 & 6 \\
\hline NCL CM Lambton Formation & -66 & 25 & 91 & & 256 & 255 & & & & 37 & 29 & 1 & & 12 & 11 & 10 & 9 \\
\hline Waratah Sandstone & 25 & 43 & 18 & & 256 & 256 & & & & 100 & & & & & & & \\
\hline WTM CM Denman Formation & 43 & 79 & 36 & & 256 & 256 & & & 19 & 53 & 28 & & & & & & \\
\hline WTM CM JP SG Mt Leonard Formatior & 79 & 124 & 46 & & 257 & 256 & & & 14 & 63 & 13 & 1 & & & & 9 & 8 \\
\hline WTM CM JP SG Althorpe Formation & 124 & 129 & 5 & & 257 & 257 & & & & & & 10 & 90 & & & & \\
\hline WTM CM JP SG Malabar Formation & 129 & 240 & 111 & & 258 & 257 & & & 5 & 53 & 22 & 6 & & & 2 & 12 & 11 \\
\hline WTM CM JP SG Mt Ogilvie Formation & 240 & 321 & 81 & & 259 & 258 & & & & 57 & 24 & 6 & & & 2 & 11 & 10 \\
\hline WTM CM JP SG Milbrodale Formation & 321 & 322 & 1 & & 259 & 259 & & & & & & & & & 100 & & \\
\hline WTM CM JP SG Mt Thorley Formation & 322 & 385 & 63 & & 259 & 259 & & & 5 & 44 & 24 & 9 & & & 4 & 14 & 13 \\
\hline WTM CM JP SG Fairford Formation & 385 & 388 & 3 & & 259 & 259 & & & & & 25 & 25 & & & 50 & & \\
\hline WTM CM JP SG Burnamwood Formati & 388 & 521 & 133 & & 260 & 259 & & & 3 & 48 & 23 & 6 & & & 2 & 18 & 17 \\
\hline WTM CM Archerfield Sandstone & 521 & 530 & 9 & & 260 & 260 & & & & 100 & & & & & & & \\
\hline WTM CM VN SG Bulga Formation & 530 & 545 & 14 & & 260 & 260 & & & & 90 & 10 & & & & & & \\
\hline WTM CM VN SG Foybrook Formation & 545 & 650 & 106 & & 261 & 260 & & & 2 & 51 & 20 & 15 & & & 2 & 10 & 9 \\
\hline
\end{tabular}

NCL CM = Newcastle Coal Measures

WTM CM = Wittingham Coal Measures

JP SG = Jerrys Plains Subgroup

VN SG = Vane Subgroup 
Pinetown 2014 Supplementary Papers

Australian Journal of Earth Sciences 61, doi: 10.1080/08120099.2014.893539

Table 2 Main model inputs for Pinegrove-1.

\begin{tabular}{|c|c|c|c|c|c|c|c|c|c|c|c|c|c|c|c|c|c|}
\hline \multirow[t]{2}{*}{ LAYER } & \multirow{2}{*}{$\begin{array}{l}\text { TOP } \\
\text { (m) }\end{array}$} & \multirow{2}{*}{$\begin{array}{l}\text { BASE } \\
\text { (m) }\end{array}$} & \multirow{2}{*}{$\begin{array}{l}\text { THICKNESS } \\
\text { (m) }\end{array}$} & \multirow{2}{*}{$\begin{array}{c}\text { EROSION } \\
\text { (m) }\end{array}$} & \multirow{2}{*}{$\begin{array}{c}\text { DEPOSITION } \\
\text { FROM } \\
\text { (Ma) }\end{array}$} & \multirow{2}{*}{$\begin{array}{c}\text { DEPOSITION } \\
\text { TO } \\
\text { (Ma) }\end{array}$} & \multirow{2}{*}{$\begin{array}{c}\text { EROSION } \\
\text { FROM } \\
\text { (Ma) }\end{array}$} & \multirow{2}{*}{$\begin{array}{c}\text { EROSION } \\
\text { TO } \\
\text { (Ma) }\end{array}$} & \multicolumn{8}{|c|}{ LITHOLOGY (\%) } & \multirow{2}{*}{$\begin{array}{l}\text { Tос } \\
\text { (\%) }\end{array}$} \\
\hline & & & & & & & & & $\begin{array}{c}\text { CONGLOM } \\
\text { ERATE }\end{array}$ & SANDSTONE & SILTSTONE & MUDSTONE & CLAYSTONE & SHALE & TUFF & COAL & \\
\hline $\begin{array}{l}\text { Late Triassic - Jurassic sediments } \\
\end{array}$ & -99 & -99 & 0 & 1075 & 205 & 135 & 130 & 90 & & 50 & & & & 50 & & & \\
\hline Wianamatta Group & -99 & -99 & 0 & 300 & 215 & 210 & 90 & 80 & & 50 & & & & 50 & & & \\
\hline Hawkesbury Sandstone & -99 & -99 & 0 & 250 & 225 & 215 & 80 & 70 & & 100 & & & & & & & \\
\hline Narrabeen Group & -99 & -99 & 0 & 800 & 252 & 225 & 70 & 10 & 50 & 50 & & & & & & & \\
\hline NCL CM Moon Island Beach Formatio & -99 & -99 & 0 & 48 & 253 & 252 & 10 & 9 & 40 & 30 & 10 & 17 & & & & 3 & 2 \\
\hline NCL CM Boolaroo Formation & -99 & -99 & 0 & 70 & 254 & 253 & 9 & 7 & & 40 & 10 & 23 & & & 10 & 17 & 15 \\
\hline NCL CM Adamstown Formation & -99 & -99 & 0 & 81 & 255 & 254 & 7 & 5 & & 45 & 15 & 28 & & & 5 & 7 & 6 \\
\hline NCL CM Lambton Formation & -99 & -99 & 0 & 91 & 256 & 255 & 5 & 3 & & 37 & 29 & 1 & & 12 & 11 & 10 & 9 \\
\hline Waratah Sandstone & -99 & -99 & 0 & 18 & 256 & 256 & 3 & 2 & & 100 & & & & & & & \\
\hline WTM CM Denman Formation & -99 & -99 & 0 & 36 & 256 & 256 & 2 & 1 & 19 & 53 & 28 & & & & & & \\
\hline WTM CM JP SG Mt Leonard Formatio & -99 & -93 & 6 & 40 & 257 & 256 & 1 & 0 & 14 & 63 & 13 & 1 & & & & 9 & 8 \\
\hline WTM CM JP SG Althorpe Formation & -93 & -92 & 1 & & 257 & 257 & & & & & & 10 & 90 & & & & \\
\hline WTM CM JP SG Malabar Formation & -92 & 96 & 188 & & 259 & 257 & & & 5 & 53 & 22 & 6 & & & 2 & 12 & 11 \\
\hline WTM CM JP SG Mt Ogilvie Formation & 96 & 109 & 13 & & 259 & 259 & & & & 42 & 30 & 12 & & & & 16 & 15 \\
\hline WTM CM JP SG Milbrodale Formatior & 109 & 131 & 22 & & 259 & 259 & & & & 60 & 35 & 5 & & & & & \\
\hline WTM CM JP SG Mt Thorley Formatior & 131 & 186 & 55 & & 259 & 259 & & & & 51 & 14 & 19 & & & 2 & 14 & 13 \\
\hline WTM CM JP SG Fairford Formation & 186 & 194 & 7 & & 260 & 259 & & & & & 25 & 25 & & & 50 & & \\
\hline WTM CM JP SG Burnamwood Format & 194 & 346 & 152 & & 261 & 260 & & & 3 & 48 & 23 & 6 & & & 2 & 18 & 17 \\
\hline WTM CM Archerfield Sandstone & 346 & 351 & 5 & & 261 & 261 & & & & 100 & & & & & & & \\
\hline WTM CM VN SG Bulga Formation & 351 & 359 & 8 & & 261 & 261 & & & & 70 & 30 & & & & & & \\
\hline WTM CM VN SG Foybrook Formation & 359 & 412 & 53 & & 262 & 261 & & & & 66 & 15 & 8 & & & 3 & 8 & 7 \\
\hline
\end{tabular}

NCL CM $=$ Newcastle Coal Measures

WTM CM = Wittingham Coal Measures

JP SG = Jerrys Plains Subgroup

VN $S G=$ Vane Subgroup 
Pinetown 2014 Supplementary Papers

Australian Journal of Earth Sciences 61, doi: 10.1080/08120099.2014.893539

Table 3 Main model inputs for DH219.

\begin{tabular}{|c|c|c|c|c|c|c|c|c|c|c|c|c|c|c|c|c|c|}
\hline \multirow[t]{2}{*}{ LAYER } & \multirow{2}{*}{$\begin{array}{l}\text { TOP } \\
\text { (m) }\end{array}$} & \multirow{2}{*}{$\begin{array}{r}\text { BASE } \\
\text { (m) }\end{array}$} & \multirow{2}{*}{$\begin{array}{c}\text { THICKNESS } \\
\text { (m) }\end{array}$} & \multirow{2}{*}{$\begin{array}{c}\text { EROSION } \\
\text { (m) }\end{array}$} & \multirow{2}{*}{$\begin{array}{l}\text { DEPOSITION } \\
\text { FROM } \\
\text { (Ma) }\end{array}$} & \multirow{2}{*}{$\begin{array}{l}\text { DEPOSITION } \\
\text { TO } \\
\text { (Ma) }\end{array}$} & \multirow{2}{*}{$\begin{array}{l}\text { EROSION } \\
\text { FROM } \\
\text { (Ma) }\end{array}$} & \multirow{2}{*}{$\begin{array}{l}\text { EROSION } \\
\text { TO } \\
\text { (Ma) }\end{array}$} & \multicolumn{8}{|c|}{ LITHOLOGY (\%) } & \multirow{2}{*}{$\begin{array}{l}\text { TOC } \\
\text { (\%) }\end{array}$} \\
\hline & & & & & & & & & $\begin{array}{c}\text { CONGLOM } \\
\text { ERATE }\end{array}$ & SANDSTONE & SILTSTONE & MUDSTONE & CLAYSTONE & SHALE & TUFF & COAL & \\
\hline Late Triassic - Jurassic sediments & -94 & -94 & 0 & 500 & 205 & 135 & 130 & 100 & & 50 & & & & 50 & & & \\
\hline Wianamatta Group & -94 & -94 & 0 & 300 & 215 & 210 & 100 & 95 & & 50 & & & & 50 & & & \\
\hline Hawkesbury Sandstone & -94 & -94 & 0 & 250 & 225 & 215 & 95 & 90 & & 100 & & & & & & & \\
\hline Narrabeen Group & -94 & -94 & 0 & 800 & 252 & 225 & 90 & 20 & 50 & 50 & & & & & & & \\
\hline NCL CM Moon Island Beach Formation & -94 & -94 & 0 & 48 & 253 & 252 & 20 & 19 & 40 & 30 & 10 & 17 & & & & 3 & 2 \\
\hline NCL CM Boolaroo Formation & -94 & -94 & 0 & 70 & 254 & 253 & 19 & 17 & & 40 & 10 & 23 & & & 10 & 17 & 15 \\
\hline NCL CM Adamstown Formation & -94 & -94 & 0 & 81 & 255 & 254 & 17 & 15 & & 45 & 15 & 28 & & & 5 & 7 & 6 \\
\hline NCL CM Lambton Formation & -94 & -94 & 0 & 28 & 255 & 255 & 15 & 14 & & 65 & 25 & & & 10 & & & \\
\hline Waratah Sandstone & -94 & -94 & 0 & 24 & 255 & 255 & 14 & 13 & & 100 & & & & & & & \\
\hline WTM CM Denman Formation & -94 & -94 & 0 & 37 & 256 & 255 & 13 & 12 & & 70 & & 30 & & & & & \\
\hline WTM CM JP SG Mt Leonard Formation & -94 & -94 & 0 & 36 & 256 & 256 & 12 & 11 & & 50 & & 34 & 3 & & & 13 & 12 \\
\hline WTM CM JP SG Althorpe Formation & -94 & -94 & 0 & 11 & 256 & 256 & 11 & 11 & & & & 10 & 90 & & & & \\
\hline WTM CM JP SG Malabar Formation & -94 & -94 & 0 & 100 & 257 & 256 & 11 & 8 & 5 & 43 & & 30 & 4 & & & 18 & 16 \\
\hline WTM CM JP SG Mt Ogilvie Formation & -94 & -94 & 0 & 139 & 258 & 257 & 8 & 4 & 1 & 56 & 2 & 29 & 5 & & & 7 & 6 \\
\hline WTM CM JP SG Milbrodale Formation & -94 & -94 & 0 & 13 & 258 & 258 & 4 & 4 & & 50 & 25 & 15 & 10 & & & & \\
\hline WTM CM JP SG Mt Thorley Formation & -94 & -94 & 0 & 99 & 259 & 258 & 4 & 2 & 2 & 40 & & 44 & 2 & & & 12 & 11 \\
\hline WTM CM JP SG Fairford Formation & -94 & -94 & 0 & 7 & 259 & 259 & 2 & 2 & & 50 & & 50 & & & & & \\
\hline WTM CM JP SG Burnamwood Formation & -94 & -30 & 64 & 98 & 261 & 259 & 2 & 0 & & 44 & 3 & 32 & 4 & & & 17 & 15 \\
\hline WTM CM Archerfield Sandstone & -30 & -24 & 6 & & 261 & 261 & & & & 100 & & & & & & & \\
\hline WTM CM VN SG Bulga Formation & -24 & -13 & 11 & & 261 & 261 & & & & 100 & & & & & & & \\
\hline WTM CM VN SG Foybrook Formation & -13 & 405 & 418 & & 264 & 261 & & & 16 & 53 & 10 & 9 & 1 & & & 11 & 10 \\
\hline
\end{tabular}

NCL CM = Newcastle Coal Measures

WTM CM = Wittingham Coal Measures

JP SG = Jerrys Plains Subgroup

VN SG = Vane Subgroup 
Pinetown 2014 Supplementary Papers

Australian Journal of Earth Sciences 61, doi: 10.1080/08120099.2014.893539

Table 4 Main model inputs for DH225.

\begin{tabular}{|c|c|c|c|c|c|c|c|c|c|c|c|c|c|c|c|c|c|}
\hline \multirow[t]{2}{*}{ LAYER } & \multirow{2}{*}{$\begin{array}{l}\text { TOP } \\
\text { (m) }\end{array}$} & \multirow{2}{*}{$\begin{array}{l}\text { BASE } \\
\text { (m) }\end{array}$} & \multirow{2}{*}{$\begin{array}{l}\text { THICKNESS } \\
\text { (m) }\end{array}$} & \multirow{2}{*}{$\begin{array}{c}\text { EROSION } \\
(\mathrm{m})\end{array}$} & \multirow{2}{*}{$\begin{array}{l}\text { DEPOSITION } \\
\text { FROM } \\
\text { (Ma) }\end{array}$} & \multirow{2}{*}{$\begin{array}{c}\text { DEPOSITION } \\
\text { TO } \\
\text { (Ma) }\end{array}$} & \multirow{2}{*}{$\begin{array}{l}\text { EROSION } \\
\text { FROM } \\
\text { (Ma) }\end{array}$} & \multirow{2}{*}{$\begin{array}{l}\text { EROSION } \\
\text { TO } \\
\text { (Ma) }\end{array}$} & \multicolumn{8}{|c|}{ LITHOLOGY (\%) } & \multirow{2}{*}{$\begin{array}{l}\text { TOC } \\
\text { (\%) }\end{array}$} \\
\hline & & & & & & & & & $\begin{array}{c}\text { CONGLOM } \\
\text { ERATE }\end{array}$ & SANDSTONE & SILTSTONE & MUDSTONE & CLAYSTONE & SHALE & TUFF & COAL & \\
\hline Late Triassic - Jurassic sediments & -102 & -102 & 0 & 200 & 205 & 135 & 130 & 100 & & 50 & & & & 50 & & & \\
\hline Wianamatta Group & -102 & -102 & 0 & 300 & 215 & 210 & 100 & 95 & & 50 & & & & 50 & & & \\
\hline Hawkesbury Sandstone & -102 & -102 & 0 & 250 & 225 & 215 & 95 & 90 & & 100 & & & & & & & \\
\hline Narrabeen Group & -102 & -102 & 0 & 800 & 252 & 225 & 90 & 20 & 50 & 50 & & & & & & & \\
\hline NCL CM Moon Island Beach Formation & -102 & -102 & 0 & 48 & 253 & 252 & 20 & 19 & 40 & 30 & 10 & 17 & & & & 3 & 2 \\
\hline NCL CM Boolaroo Formation & -102 & -102 & 0 & 70 & 254 & 253 & 19 & 17 & & 40 & 10 & 23 & & & 10 & 17 & 15 \\
\hline NCL CM Adamstown Formation & -102 & -102 & 0 & 81 & 255 & 254 & 17 & 15 & & 45 & 15 & 28 & & & 5 & 7 & 6 \\
\hline NCL CM Lambton Formation & -102 & -102 & 0 & 28 & 255 & 255 & 15 & 14 & & 65 & 25 & & & 10 & & & \\
\hline Waratah Sandstone & -102 & -102 & 0 & 24 & 255 & 255 & 14 & 13 & & 100 & & & & & & & \\
\hline WTM CM Denman Formation & -102 & -102 & 0 & 37 & 256 & 255 & 13 & 12 & & 70 & & 30 & & & & & \\
\hline WTM CM JP SG Mt Leonard Formation & -102 & -102 & 0 & 36 & 256 & 256 & 12 & 11 & & 50 & 34 & & 3 & & & 13 & 12 \\
\hline WTM CM JP SG Althorpe Formation & -102 & -102 & 0 & 11 & 256 & 256 & 11 & 11 & & & & 10 & 90 & & & & \\
\hline WTM CM JP SG Malabar Formation & -102 & -102 & 0 & 100 & 257 & 256 & 11 & 8 & 5 & 43 & & 30 & 4 & & & 18 & 16 \\
\hline WTM CM JP SG Mt Ogilvie Formation & -102 & -102 & 0 & 139 & 258 & 257 & 8 & 4 & 1 & 56 & 2 & 29 & 5 & & & 7 & 6 \\
\hline WTM CM JP SG Milbrodale Formation & -102 & -102 & 0 & 13 & 258 & 258 & 4 & 4 & & 50 & 25 & 15 & 10 & & & & \\
\hline WTM CM JP SG Mt Thorley Formation & -102 & -102 & 0 & 99 & 259 & 258 & 4 & 2 & 2 & 40 & & 44 & 2 & & & 12 & 11 \\
\hline WTM CM JP SG Fairford Formation & -102 & -102 & 0 & 7 & 259 & 259 & 2 & 2 & & 50 & & 50 & & & & & \\
\hline WTM CM JP SG Burnamwood Formation & -102 & -16 & 86 & 76 & 261 & 259 & 2 & 0 & & 59 & 21 & 13 & & & & 7 & 6 \\
\hline WTM CM Archerfield Sandstone & -16 & -7 & 9 & & 261 & 261 & & & & 100 & & & & & & & \\
\hline WTM CM VN SG Bulga Formation & -7 & 6 & 13 & & 261 & 261 & & & & 92 & 8 & & & & & & \\
\hline WTM CM VN SG Foybrook Formation & 6 & 433 & 427 & & 264 & 261 & & & 14 & 63 & 12 & 4 & & & & 7 & 6 \\
\hline
\end{tabular}

作

WTM CM = Wittingham Coal Measures

JP SG = Jerrys Plains Subgroup

VN SG = Vane Subgroup 
Pinetown 2014 Supplementary Papers

Australian Journal of Earth Sciences 61, doi: 10.1080/08120099.2014.893539

Table 5 Main model inputs for EHL-1.

\begin{tabular}{|c|c|c|c|c|c|c|c|c|c|c|c|c|c|c|c|c|c|}
\hline \multirow[t]{2}{*}{ LAYER } & \multirow{2}{*}{$\begin{array}{l}\text { TOP } \\
\text { (m) }\end{array}$} & \multirow{2}{*}{$\begin{array}{l}\text { BASE } \\
(\mathrm{m})\end{array}$} & \multirow{2}{*}{$\begin{array}{c}\text { THICKNESS } \\
\text { (m) }\end{array}$} & \multirow{2}{*}{$\begin{array}{l}\text { EROSION } \\
\qquad(\mathrm{m})\end{array}$} & \multirow{2}{*}{$\begin{array}{l}\text { DEPOSITION } \\
\text { FROM } \\
\text { (Ma) }\end{array}$} & \multirow{2}{*}{$\begin{array}{l}\text { DEPOSITION } \\
\text { TO } \\
\text { (Ma) }\end{array}$} & \multirow{2}{*}{$\begin{array}{l}\text { EROSION } \\
\text { FROM } \\
\text { (Ma) }\end{array}$} & \multirow{2}{*}{$\begin{array}{c}\text { EROSION } \\
\text { TO } \\
\text { (Ma) }\end{array}$} & \multicolumn{8}{|c|}{ LITHOLOGY (\%) } & \multirow{2}{*}{$\begin{array}{l}\text { TOC } \\
\text { (\%) }\end{array}$} \\
\hline & & & & & & & & & $\begin{array}{c}\text { CONGLOM } \\
\text { ERATE }\end{array}$ & SANDSTONE & SILTSTONE & MUDSTONE & CLAYSTONE & SHALE & TUFF & COAL & \\
\hline Late Triassic - Jurassic sediments & -170 & -170 & 0 & 1100 & 205 & 135 & 130 & 100 & & 50 & & & & 50 & & & \\
\hline Wianamatta Group & -170 & -170 & 0 & 300 & 215 & 210 & 100 & 95 & & 50 & & & & 50 & & & \\
\hline Hawkesbury Sandstone & -170 & -170 & 0 & 250 & 225 & 215 & 95 & 90 & & 100 & & & & & & & \\
\hline Narrabeen Group & -170 & -170 & 0 & 800 & 252 & 225 & 90 & 5 & 50 & 50 & & & & & & & \\
\hline NCL CM Moon Island Beach Formation & -170 & -170 & 0 & 48 & 253 & 252 & 5 & 5 & 40 & 30 & 10 & 17 & & & & 3 & 2 \\
\hline NCL CM Boolaroo Formation & -170 & -170 & 0 & 70 & 254 & 253 & 5 & 4 & & 40 & 10 & 23 & & & 10 & 17 & 15 \\
\hline NCL CM Adamstown Formation & -170 & -170 & 0 & 81 & 255 & 254 & 4 & 2 & & 45 & 15 & 28 & & & 5 & 7 & 6 \\
\hline NCL CM Lambton Formation & -170 & -170 & 0 & 28 & 255 & 255 & 2 & 1 & & 65 & 25 & & & 10 & & & \\
\hline Waratah Sandstone & -170 & -170 & 0 & 24 & 255 & 255 & 1 & 1 & & 100 & & & & & & & \\
\hline WTM CM Denman Formation & -170 & -145 & 25 & 12 & 256 & 255 & 1 & 0 & & 70 & 30 & & & & & & \\
\hline WTM CM JP SG Mt Leonard Formation & -145 & -109 & 36 & & 256 & 256 & & & & 50 & & 34 & 3 & & & 13 & 12 \\
\hline WTM CM JP SG Althorpe Formation & -109 & -98 & 11 & & 256 & 256 & & & & & & 10 & 90 & & & & \\
\hline WTM CM JP SG Malabar Formation & -98 & 1 & 100 & & 257 & 256 & & & 5 & 43 & & 30 & 4 & & & 18 & 16 \\
\hline WTM CM JP SG Mt Ogilvie Formation & 1 & 141 & 139 & & 258 & 257 & & & 1 & 56 & 2 & 29 & 5 & & & 7 & 6 \\
\hline WTM CM JP SG Milbrodale Formation & 141 & 153 & 13 & & 258 & 258 & & & & 50 & 25 & 15 & 10 & & & & \\
\hline WTM CM JP SG Mt Thorley Formation & 153 & 253 & 99 & & 259 & 258 & & & 2 & 40 & & 44 & 2 & & & 12 & 11 \\
\hline WTM CM JP SG Fairford Formation & 253 & 259 & 7 & & 259 & 259 & & & & 50 & & 50 & & & & & \\
\hline WTM CM JP SG Burnamwood Formation & 259 & 422 & 162 & & 261 & 259 & & & 4 & 40 & 3 & 32 & 4 & & & 17 & 15 \\
\hline WTM CM Archerfield Sandstone & 422 & 429 & 7 & & 261 & 261 & & & & 100 & & & & & & & \\
\hline WTM CM VN SG Bulga Formation & 429 & 429 & 0 & & 261 & 261 & & & & 50 & 25 & 15 & 10 & & & & \\
\hline WTM CM VN SG Foybrook Formation & 429 & 557 & 128 & & 262 & 261 & & & & 52 & 6 & 22 & 3 & & & 17 & 15 \\
\hline WTM CM Saltwater Creek Formation & 557 & 583 & 26 & & 262 & 262 & & & & 60 & & 40 & & & & & \\
\hline Maitland Group & 583 & 596 & 13 & & 263 & 262 & & & & & & 23 & & 77 & & & \\
\hline
\end{tabular}

NCL CM $=$ Newcastle Coal Measures

WTM CM = Wittingham Coal Measure

JP SG = Jerrys Plains Subgroup

VN SG = Vane Subgroup 
Pinetown 2014 Supplementary Papers

Australian Journal of Earth Sciences 61, doi: 10.1080/08120099.2014.893539

Table 6 Main model inputs for EHRP-1.

\begin{tabular}{|c|c|c|c|c|c|c|c|c|c|c|c|c|c|c|c|c|c|}
\hline \multirow[t]{2}{*}{ LAYER } & \multirow{2}{*}{$\begin{array}{l}\text { TOP } \\
\text { (m) }\end{array}$} & \multirow{2}{*}{$\begin{array}{l}\text { BASE } \\
\text { (m) }\end{array}$} & \multirow{2}{*}{$\begin{array}{l}\text { THICKNESS } \\
\text { (m) }\end{array}$} & \multirow{2}{*}{$\begin{array}{l}\text { EROSION } \\
\qquad(\mathrm{m})\end{array}$} & \multirow{2}{*}{$\begin{array}{l}\text { DEPOSITION } \\
\text { FROM } \\
\text { (Ma) }\end{array}$} & \multirow{2}{*}{$\begin{array}{c}\text { DEPOSITION } \\
\text { TO } \\
\text { (Ma) }\end{array}$} & \multirow{2}{*}{$\begin{array}{l}\text { EROSION } \\
\text { FROM } \\
\text { (Ma) }\end{array}$} & \multirow{2}{*}{$\begin{array}{c}\text { EROSION } \\
\text { TO } \\
\text { (Ma) }\end{array}$} & \multicolumn{8}{|c|}{ LITHOLOGY (\%) } & \multirow{2}{*}{$\begin{array}{l}\text { TOC } \\
\text { (\%) }\end{array}$} \\
\hline & & & & & & & & & $\begin{array}{l}\text { CONGLOM } \\
\text { ERATE }\end{array}$ & SANDSTONE & SILTSTONE & MUDSTONE & CLAYSTONE & SHALE & TUFF & COAL & \\
\hline Late Triassic - Jurassic sediments & -154 & -154 & 0 & 1100 & 205 & 135 & 130 & 100 & & 50 & & & & 50 & & & \\
\hline Wianamatta Group & -154 & -154 & 0 & 300 & 215 & 210 & 100 & 95 & & 50 & & & & 50 & & & \\
\hline Hawkesbury Sandstone & -154 & -154 & 0 & 250 & 225 & 215 & 95 & 90 & & 100 & & & & & & & \\
\hline Narrabeen Group & -154 & -154 & 0 & 800 & 252 & 225 & 90 & 20 & 50 & 50 & & & & & & & \\
\hline NCL CM Moon Island Beach Formation & -154 & -154 & 0 & 48 & 253 & 252 & 20 & 18 & 40 & 30 & 10 & 17 & & & & 3 & 2 \\
\hline NCL CM Boolaroo Formation & -154 & -154 & 0 & 70 & 254 & 253 & 18 & 14 & & 40 & 10 & 23 & & & 10 & 17 & 15 \\
\hline NCL CM Adamstown Formation & -154 & -154 & 0 & 81 & 255 & 254 & 14 & 10 & & 45 & 15 & 28 & & & 5 & 7 & 6 \\
\hline NCL CM Lambton Formation & -154 & -154 & 0 & 28 & 255 & 255 & 10 & 8 & & 65 & 25 & & & 10 & & & \\
\hline Waratah Sandstone & -154 & -154 & 0 & 24 & 255 & 255 & 8 & 6 & & 100 & & & & & & & \\
\hline WTM CM Denman Formation & -154 & -154 & 0 & 37 & 256 & 255 & 6 & 3 & & 70 & 30 & & & & & & \\
\hline WTM CM JP SG Mt Leonard Formation & -154 & -154 & 0 & 4 & 256 & 256 & 3 & 2 & & & & 50 & & & & 50 & 45 \\
\hline WTM CM JP SG Althorpe Formation & -154 & -154 & 0 & 1 & 256 & 256 & 2 & 1 & & & & 100 & & & & & \\
\hline WTM CM JP SG Malabar Formation & -154 & -61 & 93 & 20 & 257 & 256 & 1 & 0 & & 44 & 10 & 26 & 15 & & & 5 & 5 \\
\hline WTM CM JP SG Mt Ogilvie Formation & -61 & 48 & 109 & & 258 & 257 & & & & 40 & 10 & 37 & 5 & & & 8 & 7 \\
\hline WTM CM JP SG Milbrodale Formation & 48 & 62 & 14 & & 258 & 258 & & & & 50 & 25 & 15 & 10 & & & & \\
\hline WTM CM JP SG Mt Thorley Formation & 62 & 158 & 95 & & 259 & 258 & & & & 40 & 10 & 30 & 5 & & & 15 & 14 \\
\hline WTM CM JP SG Fairford Formation & 158 & 176 & 19 & & 259 & 259 & & & & 50 & 25 & 15 & 10 & & & & \\
\hline WTM CM JP SG Burnamwood Formation & 176 & 299 & 122 & & 260 & 259 & & & & 44 & 10 & 18 & 5 & & & 23 & 21 \\
\hline WTM CM Archerfield Sandstone & 299 & 302 & 4 & & 260 & 260 & & & & 100 & & & & & & & \\
\hline WTM CM VN SG Bulga Formation & 302 & 302 & 0 & & 260 & 260 & & & & 50 & 25 & 15 & 10 & & & & \\
\hline WTM CM VN SG Foybrook Formation & 302 & 400 & 98 & & 261 & 260 & & & & 40 & 10 & 30 & 5 & & & 15 & 14 \\
\hline WTM CM Saltwater Creek Formation & 400 & 424 & 24 & & 261 & 261 & & & & 50 & 25 & 15 & 10 & & & & \\
\hline Maitland Group & 424 & 546 & 122 & & 263 & 261 & & & & 50 & 25 & 15 & 10 & & & & \\
\hline
\end{tabular}

NCL CM $=$ Newcastle Coal Measures

WTM CM = Wittingham Coal Measures

$J P S G=$ Jerrys Plains Subgroup

VN SG = Vane Subgroup 
Pinetown 2014 Supplementary Papers

Australian Journal of Earth Sciences 61, doi: 10.1080/08120099.2014.893539

Table 7 Main model inputs for Wybong-1.

\begin{tabular}{|c|c|c|c|c|c|c|c|c|c|c|c|c|c|c|c|c|c|}
\hline \multirow[t]{2}{*}{ LAYER } & \multirow{2}{*}{$\begin{array}{l}\text { TOP } \\
\text { (m) }\end{array}$} & \multirow{2}{*}{$\begin{array}{l}\text { BASE } \\
\text { (m) }\end{array}$} & \multirow{2}{*}{$\begin{array}{c}\text { THICKNESS } \\
\text { (m) }\end{array}$} & \multirow{2}{*}{$\begin{array}{c}\text { EROSION } \\
\text { (m) }\end{array}$} & \multirow{2}{*}{$\begin{array}{l}\text { DEPOSITION } \\
\text { FROM } \\
\text { (Ma) }\end{array}$} & \multirow{2}{*}{$\begin{array}{c}\text { DEPOSITION } \\
\text { TO } \\
\text { (Ma) }\end{array}$} & \multirow{2}{*}{$\begin{array}{c}\text { EROSION } \\
\text { FROM } \\
\text { (Ma) }\end{array}$} & \multirow{2}{*}{$\begin{array}{c}\text { EROSION } \\
\text { TO } \\
\text { (Ma) }\end{array}$} & \multicolumn{8}{|c|}{ LITHOLOGY (\%) } & \multirow{2}{*}{$\begin{array}{l}\text { TOC } \\
\text { (\%) }\end{array}$} \\
\hline & & & & & & & & & $\begin{array}{c}\text { CONGLOM } \\
\text { ERATE }\end{array}$ & SANDSTONE & SILTSTONE & MUDSTONE & CLAYSTONE & SHALE & TUFF & COAL & \\
\hline Late Triassic - Jurassic sediments & -150 & -150 & 0 & 850 & 205 & 135 & 130 & 90 & & 50 & & & & 50 & & & \\
\hline Wianamatta Group & -150 & -150 & 0 & 300 & 215 & 210 & 90 & 80 & & 50 & & & & 50 & & & \\
\hline Hawkesbury Sandstone & -150 & -150 & 0 & 250 & 225 & 215 & 80 & 70 & & 100 & & & & & & & \\
\hline Narrabeen Group & -150 & -94 & 56 & 744 & 252 & 225 & 70 & 0 & 50 & 50 & & & & & & & \\
\hline NCL CM Moon Island Beach Formation & -94 & -46 & 48 & & 253 & 252 & & & 40 & 30 & 10 & 17 & & & & 3 & 2 \\
\hline NCL CM Boolaroo Formation & -46 & 24 & 70 & & 254 & 253 & & & & 40 & 10 & 23 & & & 10 & 17 & 15 \\
\hline NCL CM Adamstown Formation & 24 & 105 & 81 & & 255 & 254 & & & & 45 & 15 & 28 & & & 5 & 7 & 6 \\
\hline NCL CM Lambton Formation & 105 & 133 & 28 & & 255 & 255 & & & & 65 & 25 & & & 10 & & & \\
\hline Waratah Sandstone & 133 & 157 & 24 & & 255 & 255 & & & & 100 & & & & & & & \\
\hline WTM CM Denman Formation & 157 & 194 & 37 & & 256 & 255 & & & & 70 & 30 & & & & & & \\
\hline WTM CM JP SG Mt Leonard Formation & 194 & 197 & 4 & & 256 & 256 & & & & & & 50 & & & & 50 & 45 \\
\hline WTM CM JP SG Althorpe Formation & 197 & 199 & 1 & & 256 & 256 & & & & & & 100 & & & & & \\
\hline WTM CM JP SG Malabar Formation & 199 & 269 & 71 & & 257 & 256 & & & & 44 & 10 & 26 & 10 & & & 10 & 9 \\
\hline WTM CM JP SG Mt Ogilvie Formation & 269 & 340 & 71 & & 258 & 257 & & & & 45 & 10 & 34 & 5 & & & 6 & 6 \\
\hline WTM CM JP SG Milbrodale Formation & 340 & 340 & 0 & & 258 & 258 & & & & 70 & 30 & & & & & & \\
\hline WTM CM JP SG Mt Thorley Formation & 340 & 445 & 104 & & 259 & 258 & & & & 45 & 10 & 33 & 5 & & & 7 & 7 \\
\hline WTM CM JP SG Fairford Formation & 445 & 446 & 2 & & 259 & 259 & & & & 70 & 30 & & & & & & \\
\hline WTM CM JP SG Burnamwood Formation & 446 & 572 & 125 & & 260 & 259 & & & & 44 & 10 & 18 & 5 & & & 23 & 21 \\
\hline WTM CM Archerfield Sandstone & 572 & 573 & 1 & & 260 & 260 & & & & 100 & & & & & & & \\
\hline WTM CM VN SG Bulga Formation & 573 & 575 & 2 & & 260 & 260 & & & & 70 & 30 & & & & & & \\
\hline WTM CM VN SG Foybrook Formation & 575 & 613 & 38 & & 260 & 260 & & & & 45 & 15 & 30 & 10 & & & & \\
\hline
\end{tabular}

NCL CM $=$ Newcastle Coal Measures

WTM CM = Wittingham Coal Measure

JP SG = Jerrys Plains Subgroup

VN SG = Vane Subgroup 